\title{
Circumferential Profile Plot
}

National Cancer Institute

\section{Source}

National Cancer Institute. Circumferential Profile Plot. NCI Thesaurus. Code C94871.

A plot whose ordinate is a quantifier, such as some measure of activity concentration, at points around an annulus in a slice, and whose abscissa is in degrees. 